\title{
MICROBIAL COMPETITION FOR NUTRIENT AND WALL SITES IN PLUG FLOW*
}

\author{
DON A. JONES ${ }^{\dagger}$ AND HAL SMITH ${ }^{\dagger}$
}

\begin{abstract}
A mathematical model of microbial competition for limiting nutrients and for a limited set of available wall-attachment sites in an advection-dominated tubular reactor is formulated as a limiting case of the more general model considered in [M. Ballyk and H. L. Smith, A flow reactor with wall growth, in Mathematical Models in Medical and Health Sciences, M. Horn, G. Simonett, and G. Webb, eds., Vanderbilt University Press, Nashville, TN, 1998]. The model consists of a system of hyperbolic PDE. The existence and stability properties of its steady state solutions are investigated, both analytically and numerically. A surprising finding in the case of two-strain competition is the existence of a steady state solution characterized by the segregation of the two bacterial strains to separate nonoverlapping segments along the tubular reactor.
\end{abstract}

Key words. coexistence, random motility, uniform persistence, Sturm-Liouville eigenvalue problems

AMS subject classifications. 92B99, 35L45

PII. S0036139998341588

1. Introduction. Most mathematical models of microbial interactions in laboratory bioreactors assume that the organisms behave as planktonic cells in well-stirred solutions, ignoring their propensity to colonize surfaces and to form complex biofilms $[8,10,11]$. Exceptions include $[25,6,23]$. In natural environments and in fouled bioreactors, the environment in the biofilm is quite different than in the bulk fluid. An obvious advantage shared by wall-attached organisms is relative immunity from washout in continuous cultures or in flowing streams. Another is the tendency of trace nutrients to accumulate on surfaces providing a richer environment for the organisms. Understanding and preventing the formation of biofilm is of major economic importance in biotechnology and physiology, especially in the design of medical implants.

The role played by microbial cells attaching to human tissue surfaces is widely appreciated in various branches of physiology. Freter $[13,14,15]$ has argued that wall growth plays a major role in the observed stability of the indigenous microflora of the mammalian large intestine to colonization by invading organisms. Mathematical models, formulated by Freter et al. [13, 14, 15], strongly support this view.

In [4], a family of models of multistrain competition for a single limiting nutrient and for limited wall attachment sites in a tubular flow reactor incorporating bacterial cell motility was formulated. These models, based on those of Freter mentioned above, were largely motivated by a belief that a tubular flow reactor is a more realistic in vitro model of the colon than the chemostat. For $n$ microbial strains, the model takes the form of a system of $n+1$ coupled reaction-advection-diffusion equations for the nutrient and free-cell densities coupled to $n$ ordinary differential equations for the wall-attached strain densities. Although the single-strain case $(n=1)$ proved to be mathematically tractable (see [5]) the multistrain case poses severe difficulties for mathematical analysis. Our inability to shed much light on the multistrain model

\footnotetext{
${ }^{*}$ Received by the editors July 7, 1998; accepted for publication (in revised form) March 26, 1999; published electronically May 15, 2000.

http://www.siam.org/journals/siap/60-5/34158.html

${ }^{\dagger}$ Arizona State University, Department of Mathematics, Tempe, AZ 85287-1804 (halsmith@asu. edu). The research of the second author was supported by NSF grant DMS 9700910 .
} 
through mathematical analysis (as opposed to numerical simulations which rarely provide solid evidence of mechanisms) provides the motivation for this investigation. The main goal of this paper is to better understand multistrain bacterial competition for both limiting nutrient and for a limited number of wall-attachment sites in a tubular flow reactor.

In [5] we observed that for parameter values appropriate for the human large intestine, nutrient diffusion and bacterial motility are negligible in relation to advection. Therefore, in this paper we consider the limiting case of the model for which advection dominates diffusion and cell motility. This limiting case is called plug flow in the chemical and biological engineering literature [2]. The model reduces to a system of $2 n+1$ coupled hyperbolic PDEs. In contrast to the original model, we are able to obtain considerable information concerning the existence, uniqueness, and stability properties of its steady state solutions. The steady state equations consist of a system of differential-algebraic equations. In some cases, the steady state solutions can be shown to be asymptotically stable for the linearized system. The stability analysis is facilitated by a further reduction of the model to a system of $n$ ordinary differential equations for the wall-densities of the competing strains at the upstream end of the reactor. A global analysis of these ordinary differential equations reveals that competitive exclusion holds at the extreme upstream end of the wall of the reactor but not necessarily further downstream. In fact, a truly surprising result of this paper is the discovery of a steady state solution for two-strain competition characterized by the segregation of the two strains to separate, nonoverlapping segments of the reactor. The dominant strain occupies a segment of the nutrient-rich upstream end of the tubular reactor, excluding its rival, while the latter occupies an adjoining segment immediately downstream, essentially excluding the dominant strain. Coexistence of two populations where the populations are segregated to disjoint domains has rarely been found in mathematical models of competition. A notable exception is [22].

We begin by describing the single-strain model and showing that it results in a well-posed initial value problem. Next, sharp conditions are given, distinguishing between two dynamical regimes: washout of the organism from the reactor or the long-term survival of the strain in the form of a unique steady state solution. The multistrain model is considered next. Here, we rely on the reduction of the model to a system of ordinary differential equations for the competing strains at the upstream end of the reactor to guide our study of the existence and stability of steady state solutions. Numerical simulations illuminate our results and indicate what may occur when certain hypotheses fail. Our main results and their applications are summarized in a final discussion section. An appendix contains proofs of of our results.

2. The model for single-strain growth. In this section we formulate the model. See $[4,5]$ for more details. Consider a long thin tube extending along the $x$ axis. The reactor occupies the portion of the tube from $x=0$ to $x=L$. It is fed with a growth medium at a constant rate at $x=0$ by a laminar flow of fluid in the tube in the direction of increasing $x$ and at velocity $v$ (a constant). The external feed contains all nutrients in near optimal amounts except one, denoted by $S$, which is supplied in a constant, growth limiting concentration $S^{0}$. We allow the possibility that the feed contains bacteria at constant concentration $u^{0}$. The flow carries a medium, depleted nutrients, cells, and their byproducts out of the reactor at $x=L$. Wall-attached bacteria are assumed to be immobile. We assume negligible variation of free bacteria and nutrient concentration transverse to the axial direction of the tube.

The model accounts for the density of free bacteria (bacteria suspended in the 
fluid) $u(x, t)$, the density of wall-attached bacteria $w(x, t)$, and the density of nutrient $S(x, t)$. If $A$ denotes the cross-sectional area and $C$ the circumference of the tube, then the total free bacteria at time $t$ is given by

$$
A \int_{0}^{L} u(x, t) d x
$$

(a similar formula holds for the total nutrient) and the total bacteria on the wall at time $t$ is given by

$$
C \int_{0}^{L} w(x, t) d x
$$

The quantities $S, u$, and $w$ satisfy the following system of hyperbolic PDEs:

$$
\begin{aligned}
S_{t}+v S_{x}= & -\gamma^{-1} u f(S)-\gamma^{-1} \delta w f_{w}(S), \\
u_{t}+v u_{x}= & u(f(S)-k)+\delta w f_{w}(S)(1-G(W)) \\
& -\alpha u(1-W)+\delta \beta w, \\
w_{t}= & w\left(f_{w}(S) G(W)-k_{w}-\beta\right)+\alpha \delta^{-1} u(1-W),
\end{aligned}
$$

where $W=w / w_{\infty}$ is the occupancy fraction and $w_{\infty}$ is the maximum density of available wall sites. Boundary conditions, representing the influx of nutrient and, possibly, bacteria from the feed are given by

$$
\begin{aligned}
& v S^{0}=v S(0, t), \\
& v u^{0}=v u(0, t),
\end{aligned}
$$

and the initial conditions are

$$
S(x, 0)=S_{0}(x), \quad u(x, 0)=u_{0}(x), \quad w(x, 0)=w_{0}(x), \quad 0 \leq x \leq L .
$$

We assume that $S_{0}, u_{0}$, and $w_{0}$ are nonnegative continuous functions and that $w_{0} \leq$ $w_{\infty}$

The nutrient uptake rates for free and wall-attached bacteria are given by functions $f$ and $f_{w}$, assumed to satisfy

$$
f \in C^{1}, \quad f(0)=0, \quad f^{\prime}(S)>0 .
$$

A typical example is the Monod function

$$
f(S)=\frac{m S}{a+S} .
$$

It is assumed that there is a finite upper bound $w_{\infty}$ on the density of available wall sites for colonization. The fraction of daughter cells of wall-bound bacteria finding sites on the wall, $G(W)$, is assumed to be a decreasing function of the occupancy fraction, $W=w / w_{\infty}$. Mathematically it is assumed to satisfy

$$
G \in C^{1}, \quad 0<G(0) \leq 1, \quad G^{\prime}(W)<0, \quad G(1)=0 .
$$

Freter et al. $[13,14,15]$ use

$$
G(W)=\frac{1-W}{a+1-W},
$$


where $a$ is typically very small.

Free bacteria are attracted to the wall at a rate proportional (with constant $\alpha$ ) to the product of the free cell density $u$ and the fraction of available wall sites $1-W$. Finally, we assume that wall-attached cells are sloughed off the wall by mechanical forces proportional to their density. Except where explicitly mentioned, all parameters appearing in the model are assumed to be positive except possibly the cell death rates $k \geq 0$ and $k_{w} \geq 0$, which are sometimes ignored. See [4] for further details on the modeling.

Suitable dimensionless variables and parameters are summarized below:

$$
\begin{aligned}
\bar{S} & =S / S^{0}, \bar{u}=u / \gamma S^{0}, \bar{u}^{0}=u^{0} / \gamma S^{0}, \bar{w}=W=w / w_{\infty}, \\
\bar{x} & =x / L, \bar{t}=v t / L, \bar{f}(\bar{S})=(L / v) f\left(S^{0} \bar{S}\right), \\
\bar{f}_{w}(\bar{S}) & =(L / v) f_{w}\left(S^{0} \bar{S}\right), \bar{\alpha}=(L / v) \alpha, \bar{\beta}=(L / v) \beta, \\
\bar{k} & =(L / v) k, \bar{k}_{w}=(L / v) k_{w} .
\end{aligned}
$$

Define

$$
\epsilon=\frac{\delta w_{\infty}}{\gamma S^{0}}
$$

Then, in terms of these quantities, the model equations (2.1)-(2.2) become, on dropping the overbars,

$$
\begin{aligned}
S_{t}+S_{x}= & -u f(S)-\epsilon w f_{w}(S), \\
u_{t}+u_{x}= & u(f(S)-k)+\epsilon w f_{w}(S)(1-G(w)) \\
& -\alpha u(1-w)+\epsilon \beta w, \\
w_{t}= & w\left(f_{w}(S) G(w)-k_{w}-\beta\right)+\epsilon^{-1} \alpha u(1-w),
\end{aligned}
$$

with boundary conditions

$$
\begin{aligned}
1 & =S(0, t), \\
u^{0} & =u(0, t),
\end{aligned}
$$

and initial conditions

$$
S(x, 0)=S_{0}(x), \quad u(x, 0)=u_{0}(x), \quad w(x, 0)=w_{0}(x), \quad 0 \leq x \leq 1 .
$$

2.1. Global existences of solutions. The proof that solutions exist for a short time is a straightforward application of the method of characteristics (see, e.g., [17], but semigroup methods can also be used). However, we will require that solutions are global in time and nonnegative for $t \geq 0$. We therefore include the proof. We set $M_{1}=\sup _{x}\left(S_{0}(x), 1\right)$ and $M_{2}=\sup _{x}\left(M_{1}+u_{0}(x)+\epsilon \beta, 1+u^{0}+\epsilon \beta\right)$. We have the following a priori estimates.

Lemma 2.1. Suppose that $S(x, t) \geq 0, u(x, t) \geq 0,0 \leq w(x, t) \leq 1$ are solutions of (2.4) for $0 \leq x \leq 1, t>0$. Then

$$
\begin{aligned}
& 0 \leq S(x, t) \leq S_{0}(x-t), \\
& 0 \leq u(x, t) \leq S_{0}(x-t)+u_{0}(x-t)+\epsilon \beta, \quad 0<t<x, \\
& \\
& 0 \leq S(x, t) \leq 1, \\
& 0 \leq u(x, t) \leq 1+u^{0}+\epsilon \beta, \quad 0<x \leq t .
\end{aligned}
$$


In particular, $0 \leq S(x, t) \leq M_{1}, 0 \leq u(x, t) \leq M_{2}$ for all $0<x<1, t \geq 0$.

Proof. Let $s$ parameterize the curve $t=0,0 \leq x \leq 1$ and $x=0, t \geq 0$. Then the characteristic curves of the first two equations are given by $x=\tau+s, t=\tau$ when $t<x$ and $x=\tau, t=\tau+s$ when $t>x$. Along the characteristics we obtain from the first equations in (2.4) that

$$
\frac{d S}{d \tau}=S_{t}+S_{x} \leq 0
$$

In the case that $t<x$ we find that

$$
S(s+\tau, \tau) \leq S(s, 0)=S_{0}(s) .
$$

That is, $S(x, t) \leq S(s, 0)=S_{0}(x-t)$, which is the first inequality. If $x<t$, the same calculation shows that $S(\tau, \tau+s) \leq S(0, s)=1$. That is, $S(x, t) \leq 1$, which is the third inequality in the lemma.

The others are found in a similar fashion. In particular, the second inequality is obtained by adding the first two equations in (2.4) and estimating as before.

THEOREM 2.2. The system (2.4) has a global continuous solution (a mild solution) provided that $S_{0}(x), u_{0}(x) \geq 0,0 \leq w_{0}(x) \leq 1,0<x<1$ are continuous and that $S_{0}(0)=1, u_{0}(0)=u^{0}$.

Remark. By a mild solution we mean one that solves an integral equation associated with the integration of (2.4) along the characteristics of the PDE (see the proof in the appendix). We may also drop the compatibility conditions $S_{0}(0)=1$, $u_{0}(0)=u^{0}$ and consider discontinuous solutions to (2.4). Alternatively, we may obtain $C^{1}$ solutions to $(2.4)$ by requiring $S_{0}(x), u_{0}(x)$ to be continuously differentiable on their domains and to satisfy appropriate compatibility conditions.

3. Global stability of the washout steady state. Time-independent (steady state) solutions of $(2.4)-(2.5)$ satisfy the differential-algebraic system $\left({ }^{\prime}=\frac{d}{d x}\right)$

$$
\begin{aligned}
S^{\prime} & =-u f(S)-\epsilon w f_{w}(S), \quad S(0)=1 \\
u^{\prime} & =u(f(S)-k)+\epsilon w f_{w}(S)(1-G(w))-\alpha u(1-w)+\epsilon \beta w, \quad u(0)=u^{0}, \\
0 & =w\left(f_{w}(S) G(w)-k_{w}-\beta\right)+\epsilon^{-1} \alpha u(1-w)
\end{aligned}
$$

for $x \in[0,1]$. If the feed stream contains no bacteria $\left(u^{0}=0\right)$, which we assume throughout this section, then there is a steady state solution of (2.4)-(2.5) given by

$$
S \equiv 1, \quad u=w \equiv 0 .
$$

We call it the "washout steady state" since no bacteria are present in the reactor and, obviously, it is to be avoided if maintaining a culture is the goal.

The washout steady state is locally unique and satisfies the "stability condition"

$$
\frac{\partial H}{\partial w}(1,0,0)<0,
$$

where $H(S, u, w)$ denotes the right side of the third algebraic equation, provided that

$$
\Lambda \equiv f_{w}(1) G(0)-k_{w}-\beta<0
$$

holds. The stability condition implies that the washout steady state is asymptotically stable in the linear approximation (see Corollary 8.2). 
The main result of this section says that (3.2) is sufficient to guarantee global asymptotic stability for the washout steady state. In terms of the original parameters, the condition is that

$$
f_{w}\left(S^{0}\right) G(0)-k_{w}-\beta<0 .
$$

It says that the growth rate of wall-attached bacteria, under the conditions that wallattached cells are rare (so that a maximum fraction of daughter cells find attachment sites on the wall) and the nutrient level is the same as the nutrient level in the feed, fails to exceed the sum of the loss rates due to death and slough-off. As we shall see, it is essentially a necessary condition for global stability because when the inequality is reversed there is a nontrivial steady state and bacteria can persist on the wall.

Proposition 3.1. If $u^{0}=0$ and (3.2) holds, then every solution of (2.4)-(2.5) converges to the washout steady state, uniformly in $x \in[0,1]$.

Proof. Since $S \leq 1$ and $0 \leq w \leq 1, u$ and $w$ satisfy

$$
\begin{aligned}
u_{t}+u_{x} & \leq u(f(1)-k)+\epsilon\left(f_{w}(1)+\beta\right) w, \\
w_{t} & \leq w\left(f_{w}(1) G(0)-k_{w}-\beta\right)+\epsilon^{-1} \alpha u .
\end{aligned}
$$

The following lemma completes the proof.

Lemma 3.2. Suppose that $A, B, C, \Lambda$ are real numbers with $B, C \geq 0$. Let $u$ and $w$ be nonnegative functions satisfying

$$
\begin{aligned}
u_{t}+u_{x} & \leq A u+B w, \\
w_{t} & \leq C u+\Lambda w,
\end{aligned}
$$

with boundary condition

$$
0=u(0, t)
$$

and nonnegative initial conditions

$$
u(x, 0)=u_{0}(x), \quad w(x, 0)=w_{0}(x), \quad 0 \leq x \leq 1 .
$$

Then, for every $\eta>0$, there exists $M=M(\eta)>0$ such that

$$
u(x, t), w(x, t) \leq M \exp ((\Lambda+\eta) t) .
$$

In the next section it is shown that when $\Lambda>0$, a single-strain positive steady state solution exists.

4. Single-strain steady state and persistence. In this section we consider nontrivial steady state solutions, relaxing the assumption that $u^{0}=0$. We begin by examining the zero-set of the algebraic equation in the following lemma. The proof is given in the appendix.

LEMMA 4.1. Let

$$
H(S, u, w) \equiv w\left[f_{w}(S) G(w)-k_{w}-\beta\right]+\epsilon^{-1} \alpha u(1-w) .
$$

Then the equation $H=0$ has a unique solution $w=h(S, u) \in(0,1)$ for each $u>0$ and $0 \leq S \leq 1$. Moreover, $h(S, u)$ is continuously differentiable with $h_{u}>0$ and $h_{S}>0$. 


$$
f_{w}(1) G(0)-k_{w}-\beta<0,
$$

then $h(S, 0) \equiv 0$ extends $h$ so that $w=h(S, u)$ is the unique solution of $H=0$ and $h$ is continuously differentiable.

If

$$
f_{w}(1) G(0)-k_{w}-\beta>0,
$$

let $S^{*} \in(0,1)$ be the unique root of $f_{w}(S) G(0)-k_{w}-\beta=0$. Then $H(S, 0, w)=0$ has two branches of solutions, $w=0,0 \leq S \leq 1$ and $w=w_{*}(S)>0, S^{*}<S \leq 1$. The function $w_{*}$ is continuously differentiable and satisfies $w_{*}\left(S^{*}\right)=0, w_{*}{ }^{\prime}>0$, and $w_{*}(1)<1$. Extending $w_{*}$ by defining $w_{*}(S)=0$ for $0 \leq S \leq S^{*}$, we obtain a continuous function, not differentiable at $S=S^{*}$, with the property that $h(S, u) \rightarrow$ $w_{*}(S)$ as $u \rightarrow 0+$ and $h(S, u)>w_{*}(S)$ for $u>0$.

Our main result concerning nontrivial steady state solutions is the following theorem.

THEOREM 4.2. If either

$$
u^{0}=0 \text { and } \Lambda>0 \quad \text { or } u^{0}>0,
$$

then there is a unique steady state solution $(\bar{S}, \bar{u}, \bar{w})$ satisfying

$$
0<\bar{S}(x), \bar{u}(x), \bar{w}(x), \quad \bar{S}^{\prime}(x)<0,0<x \leq 1 .
$$

Moreover, it satisfies the stability condition

$$
\frac{\partial H}{\partial w}(\bar{S}(x), \bar{u}(x), \bar{w}(x))<0, \quad 0 \leq x \leq 1 .
$$

Consequently, the steady state is asymptotically stable in the linear approximation.

Let us explain what we mean when we say that a steady state solution is asymptotically stable in the linear approximation. As we need this idea later as well, it is convenient to digress here and give a general formulation. Consider a system of the form

$$
\begin{aligned}
X_{t}+X_{x} & =F(X, Y), \\
Y_{t} & =G(X, Y)
\end{aligned}
$$

in the strip $(x, t) \in[0,1] \times[0, \infty)$, where $X, Y$ are vectors, with boundary conditions

$$
X(0, t)=X^{0}
$$

and initial data at $t=0$. Suppose that $(\bar{X}(x), \bar{Y}(x))$ is a time-independent solution. The linearization about the steady state is

$$
\begin{aligned}
U_{t}+U_{x} & =F_{X}(\bar{X}, \bar{Y}) U+F_{Y}(\bar{X}, \bar{Y}) V, \\
V_{t} & =G_{X}(\bar{X}, \bar{Y}) U+G_{Y}(\bar{X}, \bar{Y}) V,
\end{aligned}
$$

with a homogeneous boundary condition at $x=0$. We say that $(\bar{X}, \bar{Y})$ is asymptotically stable in the linear approximation if there exist positive numbers $M, k$ such that

$$
|U(x, t)|+|V(x, t)| \leq M e^{-k t}[\|U(\bullet, 0)\|+\|V(\bullet, 0)\|], \quad t>0,
$$


where the supremum norms are used. In Corollary 8.2 we show that $(\bar{X}, \bar{Y})$ is asymptotically stable in the linear approximation if $G_{Y}(\bar{X}, \bar{Y})$ is a stable matrix for each $x \in[0,1]$.

Proof. We concentrate on the case that $u^{0}=0$ since the other case is simpler. In this case $w(0)>0$ is determined by $f_{w}(1) G(w(0))-k_{w}-\beta=0$ or, using Lemma 4.1, $w(0)=w_{*}(S=1)$. Because $\frac{\partial H}{\partial w}(1,0, w(0))=w(0) f_{w}(1) G^{\prime}(w(0))<0$, the implicit function theorem guarantees that $H=0$ can be solved for $w=h(S, u)$, satisfying $w(0)=h(1,0)$, for $(S, u)$ near $(1,0)$. On putting $w=h(S, u)$ into the first two equations of (3.1), we can solve the resulting initial value problem, at least locally. Now, $u^{\prime}(0)=\epsilon w(0)\left[f_{w}(1)(1-G(w(0)))+\beta\right]>0$ so $u(x)>0$ for small $x$. The form of the $u$-equation implies that $u(x)$ must remain positive for $x>0$ so long as it may be continued. According to Lemma 4.1, $H=0$ has a unique smooth solution $w=h(S, u)>0$ so long as $u$ is positive. Thus the solution of the differential equations for $S$ and $u$ can be solved for $0 \leq x \leq 1$. Obviously, $S^{\prime}(x)<0$; we've noted that $u(x)>0$ and $w(x)=h(S(x), u(x))>0$ for all $x$ by Lemma 4.1. Because $w$ and $u$ are positive, the third equation of (3.1) implies that $\Delta(x) \equiv f_{w}(S(x)) G(w(x))-k_{w}-\beta<0$ for $0<x \leq 1$, so

$$
\frac{\partial H}{\partial w}(S(x), u(x), w(x))=\Delta(x)+w(x) f_{w}(S(x)) G^{\prime}(w(x))-\epsilon^{-1} \alpha u(x)<0
$$

for $0 \leq x \leq 1$. Corollary 8.2 in the appendix implies the linearized stability assertion.

The case $u^{0}>0$ is simpler since $u>0$, so $w=h(S, u)>0$ is given by the first assertion of Lemma 4.1.

Information concerning the global asymptotic behavior of the wall-attached population can be obtained at $x=0$ for time-dependent solutions of (2.4)-(2.5). In view of the boundary conditions $(2.5), w(t) \equiv w(0, t)$ satisfies the ODE

$$
\frac{d w}{d t}=w\left[f_{w}(1) G(w)-k_{w}-\beta\right]+\epsilon^{-1} \alpha u^{0}(1-w) .
$$

The dynamics of (4.2) are described below.

Proposition 4.3. If condition (4.1) holds, then all positive solutions of (4.2) converge to the steady state $\bar{w}(0)>0$ (where $\bar{w}$ is defined in Theorem 4.2 ). If $u^{0}=0$ and $\Lambda \leq 0$, then all solutions of (4.2) converge to $w=0$ (as demanded by Theorem 3.1 if $\Lambda<0$ ).

Proof. It follows from Lemma 4.1 that if condition (4.1) holds, there is a unique equilibrium $w=h\left(1, u^{0}\right) \in(0,1)$ of $(4.2)$ which attracts all solutions $w(t)$ to $w(0) \in$ $[0,1]$. Obviously, $w=h\left(1, u^{0}\right)$ is precisely $\bar{w}(0)$, where $(\bar{S}(x), \bar{u}(x), \bar{w}(x))$ is the steady state solution described in Theorem 4.2. If $u^{0}=0$ and $\Lambda \leq 0$, then $w^{\prime}(t)<0$ for any positive solution so $w(t)$ converges as $t \rightarrow \infty$ and $w=0$ is the only steady state.

Proposition 4.3 suggests that the steady state, described in Theorem 4.2, may be globally attracting and it confirms that the conditions of Theorem 4.2, namely (4.1), are sufficient to guarantee that the bacteria population can persist in the reactor, at least on the wall near the upstream end. In addition, we see that a simple ODE appears to mimic faithfully the dynamics of the hyperbolic system. This observation motivates our analysis of the multistrain system.

5. Multistrain competition. The model of exploitative competition between $n$ bacterial strains for a single nutrient in the plug flow reactor in which each strain 
competes for a common set of sites on the wall is given by (see [4])

$$
\begin{aligned}
S_{t}+v S_{x}= & -\sum_{i=1}^{n} \gamma_{i}^{-1}\left[u_{i} f_{u_{i}}(S)+\delta w_{i} f_{w_{i}}(S)\right] \\
\left(u_{i}\right)_{t}+v\left(u_{i}\right)_{x}= & u_{i}\left(f_{u_{i}}(S)-k_{u_{i}}\right)+\delta w_{i} f_{w_{i}}(S)\left(1-G_{i}(W)\right) \\
& -\alpha_{i} u_{i}(1-W)+\delta \beta_{i} w_{i} \\
\left(w_{i}\right)_{t}= & w_{i} f_{w_{i}}(S) G_{i}(W)-k_{w_{i}} w_{i}+\delta^{-1} \alpha_{i} u_{i}(1-W)-\beta_{i} w_{i},
\end{aligned}
$$

where

$$
W(x, t)=\left(\sum_{i=1}^{n} w_{i}(x, t)\right) / w_{\infty}
$$

is the occupation fraction. The boundary conditions are given by

$$
\begin{aligned}
v S(0, t) & =v S^{0}, \\
v u_{i}(0, t) & =v u_{i}^{0},
\end{aligned}
$$

and the initial conditions are

$$
S(x, 0)=S_{0}(x), u_{i}(x, 0)=u_{i 0}(x), w_{i}(x, 0)=w_{i 0}(x) .
$$

All initial conditions are assumed to be nonnegative and $\sum_{i=1}^{n} w_{i 0} \leq w_{\infty}$

The equations can be simplified by nondimensionalizing the parameters, dependent, and independent variables exactly as before. The scaled version is then

$$
\begin{aligned}
S_{t}+S_{x}= & -\sum_{i=1}^{n}\left[u_{i} f_{u_{i}}(S)+\epsilon_{i} w_{i} f_{w_{i}}(S)\right], \\
\left(u_{i}\right)_{t}+\left(u_{i}\right)_{x}= & u_{i}\left(f_{u_{i}}(S)-k_{u_{i}}\right)+\epsilon_{i} w_{i} f_{w_{i}}(S)\left(1-G_{i}(W)\right) \\
& -\alpha_{i} u_{i}(1-W)+\epsilon_{i} \beta_{i} w_{i}, \\
\left(w_{i}\right)_{t}= & w_{i} f_{w_{i}}(S) G_{i}(W)-k_{w_{i}} w_{i}+\epsilon_{i}^{-1} \alpha_{i} u_{i}(1-W)-\beta_{i} w_{i},
\end{aligned}
$$

with boundary conditions

$$
S(0, t)=1, \quad u_{i}(0, t)=u_{i}^{0},
$$

and initial conditions

$$
S(x, 0)=S_{0}(x), u_{i}(x, 0)=u_{i 0}(x), w_{i}(x, 0)=w_{i 0}(x) .
$$

The initial data $S_{0}, u_{i 0}$, and $w_{i 0}$ are continuous and nonnegative and satisfy

$$
\sum_{i=1}^{n} w_{i 0}(x) \leq 1
$$

We make no attempt to analyze this complicated time-dependent system (wellposedness can be treated in a similar manner as for one strain). We will be interested in stable steady state solutions of (5.1) and in the system of ODE for $w_{i}(0, t)$ obtained by inserting the boundary conditions (5.2) for $S$ and $u_{i}$ (recall (4.2)). 
Steady state solutions satisfy the following differential-algebraic system for $x \in$ $[0,1]$ :

$$
\begin{aligned}
S^{\prime}= & -\sum_{i=1}^{n}\left[u_{i} f_{u_{i}}(S)+\epsilon_{i} w_{i} f_{w_{i}}(S)\right], \quad S(0)=1, \\
u_{i}^{\prime}= & u_{i}\left(f_{u_{i}}(S)-k_{u_{i}}\right)+\epsilon_{i} w_{i} f_{w_{i}}(S)\left(1-G_{i}(W)\right) \\
& -\alpha_{i} u_{i}(1-W)+\epsilon_{i} \beta_{i} w_{i}, \quad u_{i}(0)=u_{i}^{0}, \\
0= & w_{i} f_{w_{i}}(S) G_{i}(W)-k_{w_{i}} w_{i}+\epsilon_{i}^{-1} \alpha_{i} u_{i}(1-W)-\beta_{i} w_{i} .
\end{aligned}
$$

These steady state equations have the general form

$$
\begin{aligned}
y^{\prime} & =F(y, w), \quad y(0)=y_{0}, \\
0 & =H(y, w),
\end{aligned}
$$

where $F$ and $H$ are continuously differentiable. The component $y$ of a solution $(y(x), w(x))$ on an interval $I$ of this system will be continuously differentiable on $I$ while $w$ will be at least continuous. If

$$
\operatorname{det} \frac{\partial H}{\partial w}(y(x), w(x)) \neq 0, \quad x \in I
$$

then the solution is locally unique in the sense that any other solution which agrees with it at one point agrees with it on the intersection of their domains (implicit function theorem). In this case, $w$ is continuously differentiable by the implicit function theorem. We say that the solution satisfies the "stability condition" if $\frac{\partial H}{\partial w}(y(x), w(x))$ is a stable matrix, i.e., all its eigenvalues have a negative real part.

Taking inspiration from the fact that the dynamics of the ODE (4.2) for the wall density at $x=0$ appear to give a faithful representation of the dynamics of the full model in the single-strain case (see Proposition 4.3), we are motivated to make a similar study for multistrain competition. Set $w_{i}(t)=w_{i}(0, t)$ and use the boundary conditions to get

$$
\frac{d w_{i}}{d t}=w_{i}\left[f_{w_{i}}(1) G_{i}(W)-k_{w_{i}}-\beta_{i}\right]+\epsilon^{-1} \alpha_{i} u_{i}^{0}(1-W),
$$

where $W=\sum_{i=1}^{n} w_{i}$. Equations (5.5) define a dynamical system on

$$
\Delta=\left\{w \in \mathbb{R}^{n}: w_{i} \geq 0, \sum_{i=1}^{n} w_{i} \leq 1\right\} .
$$

In order to keep things as simple as possible, we focus primarily on the case of two strains $(n=2)$. In this case, $(5.5)$ is a competitive planar system so all solutions converge to an equilibrium (e.g., see Theorem 2.2 in [24]). We will consider two representative cases below.

The reduction of the hyperbolic system to the ODE (5.5) appears to be justified from a biological point of view. The upstream end of the reactor $(x=0)$ is clearly the richest in terms of nutrient availability so an organism that cannot survive on the wall at $x=0$ is most likely relegated to a subordinate role. From a mathematical point of view it should be remembered that this reduction does give the true values of the wall-attached cell densities at $x=0$ so in this sense it is not an approximation, although it may not provide the whole story. Numerical simulations can be used to verify the predictions of the reduced system. 
5.1. Bacteria-free feed stream: $\boldsymbol{u}_{\boldsymbol{i}}^{0}=0$. In this case, the equations (5.5) simplify considerably $(n=2)$ :

$$
\frac{d w_{i}}{d t}=w_{i}\left[f_{w_{i}}(1) G_{i}\left(w_{1}+w_{2}\right)-k_{w_{i}}-\beta_{i}\right]
$$

In particular, the wall-affinity parameters, $\alpha_{i}$, play no role in determining the outcome of competition between wall-attached cells at $x=0$. We assume that both competitors are viable,

$$
f_{w_{i}}(1) G_{i}(0)-k_{w_{i}}-\beta_{i}>0, \quad i=1,2,
$$

so that, in addition to the trivial steady state $(0,0)$ of $(5.6)$ corresponding to the washout steady state $\left(S, u_{1}, u_{2}, w_{1}, w_{2}\right) \equiv(1,0,0,0,0)$ of $(5.4)$, the single-strain steady states $\left(w_{1}^{*}, 0\right)$ and $\left(0, w_{2}^{*}\right)$ exist. The occupancy fractions $w_{i}^{*}$ for these single-strain steady states satisfy

$$
w_{i}^{*}=G_{i}^{-1}\left(\frac{k_{w_{i}}+\beta_{i}}{f_{w_{i}}(1)}\right) \text {. }
$$

As $u_{i}^{0}=0$ and (5.7) hold, Theorem 4.2 implies that (5.4) has the single-strain steady state solutions

$$
E_{1}=\left(S, u_{1}, u_{2}, w_{1}, w_{2}\right)=\left(\bar{S}(x), \bar{u}_{1}(x), 0, \bar{w}_{1}(x), 0\right)
$$

and

$$
E_{2}=\left(S, u_{1}, u_{2}, w_{1}, w_{2}\right)=\left(\hat{S}(x), 0, \hat{u}_{2}(x), 0, \hat{w}_{2}(x)\right) .
$$

Note that $w_{1}^{*}=\bar{w}_{1}(0)$ and $w_{2}^{*}=\hat{w}_{2}(0)$.

We will ignore the nongeneric case of equality of the $w_{i}^{*}$ and assume, by renumbering if necessary, that

$$
w_{1}^{*}>w_{2}^{*}
$$

Then, there are no positive steady states $\left(w_{1}, w_{2}\right)$ of $(5.6)$ because any such steady state must satisfy $w_{1}+w_{2}=w_{i}^{*}, i=1,2$, which is clearly impossible. In fact, strain one is the winner at the upstream end.

TheOREM 5.1. Suppose that (5.7) and $w_{1}^{*}>w_{2}^{*}$ hold. Then all solutions $\left(w_{1}(t), w_{2}(t)\right)$ of $(5.6)$ satisfying $w_{1}(0)>0$ converge to $\left(w_{1}^{*}, 0\right)$.

Proof. We have already noted above that all solutions converge to a steady state and that there are exactly three of these. The "invasion eigenvalue" of the variational equation about $\left(w_{1}^{*}, 0\right)$ is given by

$$
\lambda_{12}=f_{w_{2}}(1) G_{2}\left(w_{1}^{*}\right)-k_{w_{2}}-\beta_{2} .
$$

As $f_{w_{2}}(1) G_{2}\left(w_{2}^{*}\right)-k_{w_{2}}-\beta_{2}=0, w_{1}^{*}>w_{2}^{*}$, and $G_{2}$ is strictly decreasing, it follows that $\lambda_{12}$ is negative. An analogous calculation shows that $\left(0, w_{2}^{*}\right)$ is unstable to invasion by strain one. The result now follows from elementary phase plane analysis or by Theorem B of [16].

When the feed stream contains no bacteria, the winning strain $($ at $x=0)$ is the one whose occupancy fraction (in the absence of its competitor) is largest. We see 
from (5.8) that the death and slough-off rates as well as the growth rate come in to play in determining a good competitor.

We remark that Theorem 5.1 continues to hold if (5.7) is replaced by $f_{w_{i}}(1) G_{i}(0)-$ $k_{w_{i}}-\beta_{i}>0$ for $i=1$ only ( $w_{2}^{*}$ may not exist). In fact, using a Lyapunov function approach, the above analysis can be extended to $n$ competing strains if $k_{w_{i}}+\beta_{i}$ are independent of $i$. For this case, define $w_{i}^{*}$ as in (5.8) if $\frac{k_{w_{i}}+\beta_{i}}{f_{w_{i}}(1)}$ belongs to the range of $G_{i}$ (note its domain is $[0,1]$ ) and define $w_{i}^{*}=0$ otherwise. By renumbering we assume that

$$
w_{n}^{*} \leq w_{n-1}^{*} \leq \cdots \leq w_{2}^{*}<w_{1}^{*} .
$$

(Note the last inequality is assumed to be strict!) Arguing as in Theorem 3.2 of [26], we find that every solution of the $n$ strain analogue of $(5.6)$ with $w_{1}(0)>0$ converges to the steady state with $w_{1}=w_{1}^{*}$ and $w_{i}=0$ for $i \neq 1$.

Returning to the case $n=2$, Theorem 5.1 implies that if we seek stable steady state solutions $\left(S, u_{1}, u_{2}, w_{1}, w_{2}\right)$ of $(5.1)$, then we need only consider the case that

$$
w_{1}(0)=w_{1}^{*}=\bar{w}_{1}(0), w_{2}(0)=0 .
$$

However, it does not necessarily imply that $E_{1}$ is the only such steady state (since $E_{1}$ may not be locally unique) or even that it is stable. In fact, it turns out that any stable steady state solution must agree with $E_{1}$ near $x=0$.

Proposition 5.2. Suppose that (5.7) and $w_{1}^{*}>w_{2}^{*}$ hold. If $\left(S, u_{1}, u_{2}, w_{1}, w_{2}\right)$ is a solution of (5.4) satisfying

$$
w_{1}(0)=w_{1}^{*}=\bar{w}_{1}(0), w_{2}(0)=0,
$$

then there exists $x_{0}>0$ such that

$$
\left(S(x), u_{1}(x), u_{2}(x), w_{1}(x), w_{2}(x)\right)=\left(\bar{S}(x), \bar{u}_{1}(x), 0, \bar{w}_{1}(x), 0\right)
$$

for $0 \leq x \leq x_{0}$. Moreover, $\left(S, u_{1}, u_{2}, w_{1}, w_{2}\right)$ satisfies the stability condition on this interval. The value of $x_{0}$ is determined by the requirement

$$
f_{w_{2}}(\bar{S}(x)) G_{2}\left(\bar{w}_{1}(x)\right)-k_{w_{2}}-\beta_{2}<0, \quad 0 \leq x<x_{0} .
$$

Proof. Let $H=\left(H_{1}, H_{2}\right)$ denote the right-hand side of the last two equations of (5.4) so that they can be written as $H=0$. By the implicit function theorem, it suffices to show that $E_{1}$ satisfies the stability condition for small $x$. The relevant Jacobian, evaluated along the steady state $E_{1}$, is

$$
\frac{\partial H}{\partial w}(x)=\left(\begin{array}{cc}
\Delta_{1}+\bar{w}_{1} f_{w_{1}}(\bar{S}) G_{1}^{\prime}\left(\bar{w}_{1}\right)-\epsilon_{1}^{-1} \alpha_{1} \bar{u}_{1} & \bar{w}_{1} f_{w_{1}}(\bar{S}) G_{1}^{\prime}\left(\bar{w}_{1}\right)-\epsilon_{1}^{-1} \alpha_{1} \bar{u}_{1} \\
0 & \Delta_{2},
\end{array}\right),
$$

where the argument $x$ has been suppressed and

$$
\Delta_{i}=f_{w_{i}}(\bar{S}) G_{i}\left(\bar{w}_{1}\right)-k_{w_{i}}-\beta_{i} .
$$

At $x=0, \Delta_{1}=f_{w_{1}}(1) G_{1}\left(w_{1}^{*}\right)-k_{w_{1}}-\beta_{1}=0$ and $\Delta_{2}=f_{w_{2}}(1) G_{2}\left(w_{1}^{*}\right)-k_{w_{2}}-\beta_{2}<0$, the latter by virtue of our assumption $w_{1}^{*}>w_{2}^{*}$ and the monotonicity of $G_{2}$. As $\bar{w}_{1}(0)=w_{1}^{*}>0$ and $G_{i}^{\prime}<0$, we see that the Jacobian is stable at $x=0$. We argued that $\Delta_{1}(x)<0$ for $0<x \leq 1$ in Theorem 4.2. Thus, the Jacobian is a stable matrix so long as $\Delta_{2}(x)<0$, which includes a neighborhood of $x=0$ by continuity. 
Assuming that solutions of (5.1) approach steady state, we may conclude from Theorem 5.1 and Proposition 5.2 that $w_{1}^{*}>w_{2}^{*}$ implies strain one dominates the upstream end of the flow reactor in the sense that strain two is absent near $x=0$. However, it may not be absent from the wall altogether (see Figure 6.3).

It is natural to seek additional conditions implying that $E_{1}$ is locally unique (no other can branch off it) and asymptotically stable in the linear approximation (see the definition in section 4 ). If this holds, then $E_{1}$ is the unique stable steady state of (5.4) by Theorem 5.1 and the local uniqueness. The following result, giving such conditions, is a corollary of the proof of Proposition 5.2. We begin by establishing some convenient notation. Recall from Lemma 4.1 that (5.7) implies that the equation

$$
f_{w_{i}}(S) G_{i}(W)-k_{w_{i}}-\beta_{i}=0
$$

can be solved for the strictly increasing function

$$
W=W_{i *}(S)=G_{i}^{-1}\left(\frac{k_{w_{i}}+\beta_{i}}{f_{w_{i}}(S)}\right)
$$

for $S_{i *} \leq S \leq 1$, where $f_{w_{i}}\left(S_{i *}\right) G_{i}(0)-k_{w_{i}}-\beta_{i}=0$. Indeed, $W_{i *}(1)=w_{i}^{*}$ for $i=1,2$. Thus, our assumption $w_{1}^{*}>w_{2}^{*}$ implies that, at least for $S$ near $S=1$, $W_{1 *}(S)>W_{2 *}(S)$. It is convenient in stating our hypotheses to extend the definition of $W_{i *}(S)$ to all of $0 \leq S \leq 1$ by setting it to be zero on $\left[0, S_{i *}\right)$. This results in a continuous function, differentiable except at $S=S_{i *}$ (see Lemma 4.1).

Corollary 5.3. Suppose that $W_{1 *}(S) \geq W_{2 *}(S)$ for $0 \leq S \leq 1$. Then the singlestrain steady state $E_{1}$ is a locally unique solution of (5.4) and it is asymptotically stable in the linear approximation.

Proof. From the proof of Proposition 5.2, it suffices to show that $\Delta_{2}(x)<0$ for all $x \in[0,1]$. From Lemma 4.1, we have that $\bar{w}_{1}(x)=h\left(\bar{S}(x), \bar{u}_{1}(x)\right)>W_{1 *}(S(x))$. (Actually, in the lemma we used the notation $w_{*}$ for what we now call $W_{1 *}$.) Therefore, our assumption implies that $\bar{w}_{1}(x)>W_{2 *}(S(x))$ so that

$$
\begin{aligned}
\Delta_{2}(x) & =f_{w_{2}}(\bar{S}(x)) G_{2}\left(\bar{w}_{1}(x)\right)-k_{w_{2}}-\beta_{2} \\
& <f_{w_{2}}(\bar{S}(x)) G_{2}\left(W_{2 *}(\bar{S}(x))\right)-k_{w_{2}}-\beta_{2} \\
& =0
\end{aligned}
$$

for $x \in[0,1]$. Corollary 8.2 implies the linearized stability assertion. This completes our proof.

An interesting case occurs when the two curves $W_{i *}(S)$ cross. In that case, the steady state $E_{1}$ may not satisfy the stability condition for all $x$ and, in fact, the steady state $E_{2}$ may satisfy the stability condition for an interval of $x$ values. For definiteness, assume that there is an $S_{0}$ such that $W_{1 *}(S)<W_{2 *}(S)$ for $S<S_{0}$. Consider the stability of steady state $E_{2}$. Assume that $\hat{S}(x)<S_{0}$ for $x_{1}<x \leq 1$ (recall that it is strictly decreasing), then $\hat{w}_{2}(x)=h\left(\hat{S}(x), \hat{u}_{2}(x)\right)>W_{2 *}(\hat{S}(x)) \geq W_{1 *}(\hat{S}(x))$ for $x_{1}<x \leq 1$, so in the Jacobian evaluated along $E_{2}$ we have

$$
\begin{aligned}
\Delta_{1}(x) & =f_{w_{1}}(\hat{S}(x)) G_{1}\left(\hat{w}_{2}(x)\right)-k_{w_{1}}-\beta_{1} \\
& <f_{w_{1}}(\hat{S}(x)) G_{1}\left(W_{1 *}(x)\right)-k_{w_{1}}-\beta_{1} \\
& =0 .
\end{aligned}
$$

Hence $\left(\Delta_{2}(x)<0\right.$ follows easily), $E_{2}$ satisfies the stability condition for $x_{1}<x \leq 1$. In this case, we might expect the existence of a steady state which near $x=0$ agrees with $E_{1}$, while near $x=1$ it agrees with $E_{2}$. Our numerical simulations confirm this expectation. 
5.2. Bacteria in the feed stream: $\boldsymbol{u}_{\boldsymbol{i}}^{\mathbf{0}}>\mathbf{0}$. We assume that $u_{i}^{0}>0$ for $i=1,2$. In this case, there are no steady states of (5.5) on the coordinate axes: the vector field points inward everywhere on the boundary of $\Delta$. However, there exists a unique coexistence steady state for (5.5). To see this, first observe that if $\left(\hat{w}_{1}, \hat{w}_{2}\right)$ is a steady state of (5.5) with $\hat{w}_{i}>0$, then we must have $f_{w_{i}}(1) G_{i}(W)-k_{w_{i}}-\beta_{i}<0$, where $W=\hat{w}_{1}+\hat{w}_{2}$. Solving for $\hat{w}_{i}$ in terms of $W$ we get

$$
\hat{w}_{i}=\frac{\epsilon_{i}^{-1} \alpha_{i} u_{i}^{0}(1-W)}{k_{w_{i}}+\beta_{i}-f_{w_{i}}(1) G_{i}(W)},
$$

and adding gives a single equation for $W$ :

$$
\frac{W}{1-W}=\frac{\epsilon_{1}^{-1} \alpha_{1} u_{1}^{0}}{k_{w_{1}}+\beta_{1}-f_{w_{1}}(1) G_{1}(W)}+\frac{\epsilon_{2}^{-1} \alpha_{2} u_{2}^{0}}{k_{w_{2}}+\beta_{2}-f_{w_{2}}(1) G_{2}(W)} .
$$

The function on the left increases without bound as $W$ nears one while the function on the left is positive and strictly decreasing on its domain (where both denominators are positive). Thus (5.10) has a unique root $\widehat{W}$ satisfying $0<\widehat{W}<1$. Equation (5.9) determines the values of $w_{i}$. Clearly, the resulting steady state $\left(\hat{w}_{1}, \hat{w}_{2}\right)$ is unique and we show that it attracts all solutions. Thus, coexistence of the two strains occurs if $u_{i}^{0}>0$.

THEOREM 5.4. If $u_{i}^{0}>0$ for $i=1,2$, then every solution of (5.5) converges to $\left(\hat{w}_{1}, \hat{w}_{2}\right)$.

Proof. It can be shown that $\left(\hat{w}_{1}, \hat{w}_{2}\right)$ is asymptotically stable in the linear approximation. However, it is unnecessary to check this because every solution must converge to an equilibrium and it is the only one.

We expect that there exists a unique steady state solution $\left(S, u_{1}, u_{2}, w_{1}, w_{2}\right)$ of (5.4) satisfying $w_{i}(0)=\hat{w}_{i}$ for $i=1,2$ which is asymptotically stable in the linear approximation. Our next result asserts just that.

THEOREM 5.5. If $u_{i}^{0}>0$ for $i=1,2$, then there exists a unique steady state solution $\left(S^{*}, u_{1}^{*}, u_{2}^{*}, w_{1}^{*}, w_{2}^{*}\right)$ of (5.4) satisfying $w_{i}^{*}(0)=\hat{w}_{i}$ for $i=1,2$. Moreover, it satisfies

$$
u_{i}^{*}(x)>0, w_{i}^{*}(x)>0, \quad x \in[0,1],
$$

and it is asymptotically stable in the linear approximation.

Proof. The arguments used to produce the steady state $\left(\hat{w}_{1}, \hat{w}_{2}\right)$ of $(5.5)$ can be used to solve the algebraic part $H=0$ of $(5.4)$ for $\left(w_{1}, w_{2}\right)$ as a smooth function, $\left(w_{1}, w_{2}\right)=G\left(S, u_{1}, u_{2}\right)$ when $u_{i}>0$ for $i=1,2$ and we have $\Delta_{i}=f_{w_{i}}(S) G_{i}(W)-$ $k_{w_{i}}-\beta_{i}<0$, where $W=w_{1}+w_{2}$ and $w_{i}>0$. The Jacobian $\frac{\partial H}{\partial w}$, along $w=G(S, u)$, is

$$
\left(\begin{array}{cc}
\Delta_{1}+w_{1} f_{w_{1}}(S) G_{1}^{\prime}\left(w_{1}\right)-\epsilon_{1}^{-1} \alpha_{1} u_{1}, & w_{1} f_{w_{1}}(S) G_{1}^{\prime}\left(w_{1}\right)-\epsilon_{1}^{-1} \alpha_{1} u_{1} \\
w_{2} f_{w_{2}}(S) G_{2}^{\prime}\left(w_{2}\right)-\epsilon_{2}^{-1} \alpha_{2} u_{2}, & \Delta_{2}+w_{2} f_{w_{2}}(S) G_{2}^{\prime}\left(w_{2}\right)-\epsilon_{2}^{-1} \alpha_{2} u_{2}
\end{array}\right) .
$$

Hence

$$
\begin{aligned}
\operatorname{det} \frac{\partial H}{\partial w}= & \Delta_{1} \Delta_{2}+\left[w_{2} f_{w_{2}}(S) G_{2}^{\prime}\left(w_{2}\right)-\epsilon_{2}^{-1} \alpha_{2} u_{2}\right] \Delta_{1} \\
& +\left[w_{1} f_{w_{1}}(S) G_{1}^{\prime}\left(w_{1}\right)-\epsilon_{1}^{-1} \alpha_{1} u_{1}\right] \Delta_{2}>0
\end{aligned}
$$

and its trace is negative. Thus the Jacobian is a stable matrix. Corollary 8.2 guarantees the linearized stability. Inserting $\left(w_{1}, w_{2}\right)=G\left(S, u_{1}, u_{2}\right)$ into the ODEs for $S, u_{1}, u_{2}$, we obtain a well-posed initial value problem. The initial conditions and the form of the differential equations for $u_{i}$ ensure that $u_{i}(x)$ remains positive. 


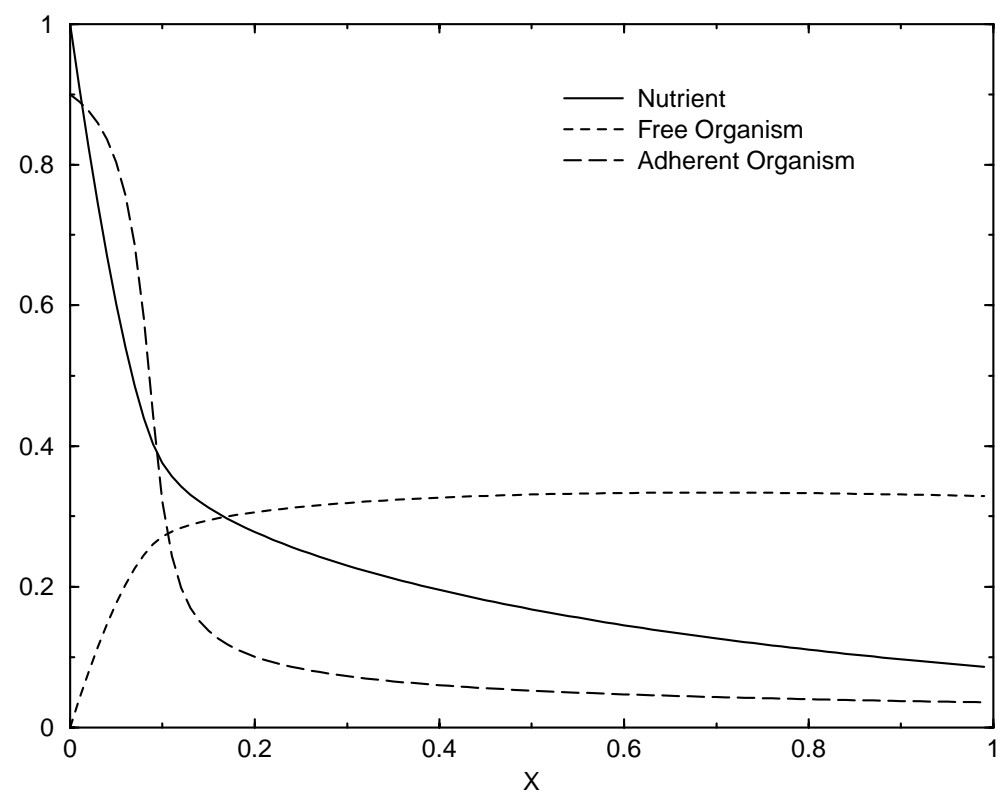

FIG. 6.1. Steady state solution for the single species run when $u^{0}=0$ and $\Lambda>0$.

6. Numerics. The goal of this section is to present the results of some numerical simulations which complement the analytic results of the previous sections. We make no claim that the parameters we use are biologically reasonable.

To approximate the solutions of (2.4) we discretize the family of ODEs given by (8.1). In particular, we use a forward Euler time discretization of the PDE (2.4) along its characteristics. In all simulations we uniformly partition the interval $0 \leq x \leq 1$ into 100 cells and we choose $L=1, S^{0}=1$. Moreover, in the two-strain simulations we always choose $f_{u_{i}}(S)=f_{w_{i}}(S)=m_{i} S /\left(a_{i}+S\right), i=1,2, G_{1}(w)=G_{2}(w)=$ $(1-w) /(1.1-w)$. The one-strain simulations use $G(w)=G_{1}(w)$. Steady states are computed by running our approximation to a steady state. This means that to the accuracy of our computer, iterates of our discretization do not change. All simulations resulted in convergence to equilibrium.

In Figure 6.1 we illustrate the form of a steady state solution of a single strain simulation in a bacteria-free stream $\left(u^{0}=0\right)$. We choose $f(S)=f_{w}(S)=S /(1+S)$, $k_{w}=.05$, and $\beta=.2$. Thus $\Lambda=f_{w}(1) G(0)-k_{w}-\beta>0$, and Theorem 4.2 indicates that there is a unique positive steady state. The other parameters are $\alpha=.2, \epsilon=10.0$, $k=.2$. Changing these parameters only affects the shape of the positive steady state (see Figure 6.2).

In Figure 6.2 we consider a two-strain simulation in a bacteria-free feed stream. We let $k_{w_{1}}=k_{w_{2}}=.05, \beta_{1}=\beta_{2}=.2, f_{w_{1}}(S)=f_{w_{2}}(S)=S /(1+S)$. Thus $w_{1}^{*}=w_{2}^{*}$ and this is the nongeneric case that we did not consider in the analysis. This example is chosen to illustrate the effects of the other parameters. For example, a larger value of $k_{i}$ will decrease the density of the free organism for increasing $x$ and increase the density of the nutrient. Smaller $\alpha_{i}$ will diminish the density of the wall growth away from the upstream end. Decreasing $\epsilon_{i}$ will diminish the presence of the free strain. The attributes are expected, and we found no parameters that would exclude a competitor $\left(\right.$ with $\left.w_{1}^{*}=w_{2}^{*}\right)$. 

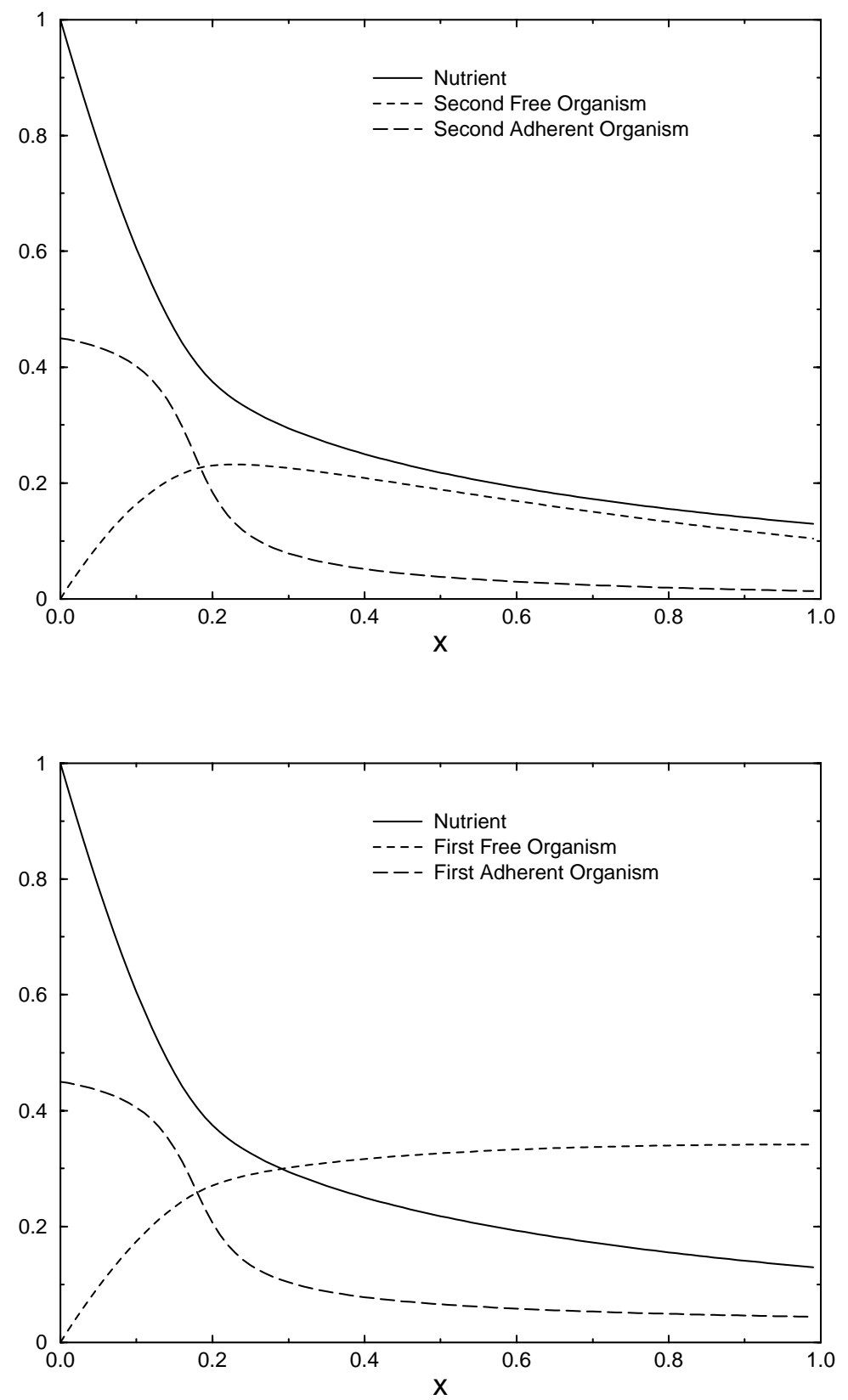

FIG. 6.2. Steady state solution of a two-strain competition with $w_{1}^{*}=w_{2}^{*}, u_{i}^{0}=0, i=1,2$. The other parameters are $k_{1}=1.5, k_{2}=.2, \alpha_{1}=\alpha_{2}=.2, \beta_{1}=\beta_{2}=.2, \epsilon_{1}=\epsilon_{2}=10.0$. The latter parameters affect only the shape of the densities and not the stability.

An example showing that $w_{1}^{*}>w_{2}^{*}$ does not imply the demise of the second strain is presented in Figure 6.3. We chose the uptake function $f_{u_{1}}(S)=3 S /(2+S)$ and $f_{u_{2}}(S)=S /(.33+S)$. Thus for $S \geq 1 / 2$ the first strain has the advantage, and strain two has the advantage for $S \leq 1 / 2$. In particular, the functions $W_{1 *}(S)$ and $W_{2 *}(S)$ cross. The other parameters are $k_{w_{1}}=k_{w_{2}}=0, k_{1}=k_{2}=0, \alpha_{1}=\alpha_{2}=.2$, 

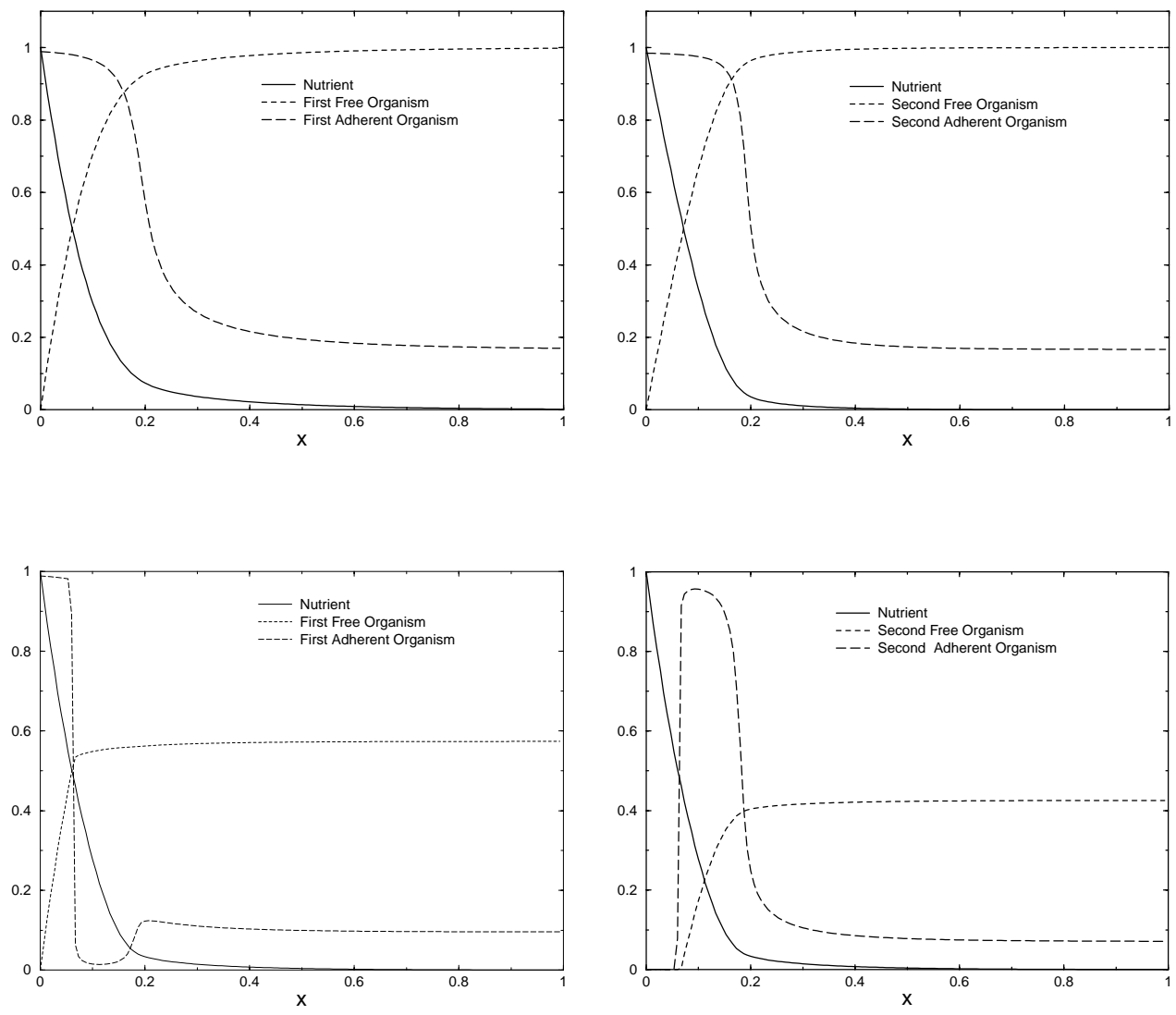

FIG. 6.3. Steady state solution of a two-strain competition with $w_{1}^{*}>w_{2}^{*}$ and $u_{i}^{0}=0, i=1,2$. The lower figures show the steady state solution in the absence of the other species. Notice $w_{2}(x)=$ $0=u_{2}(x)$ and $S(x), w_{1}(x), u_{2}(x)$ agree with the steady state of $S, u_{1}, w_{1}$ in the absence of $u_{2}, w_{2}$ (lower left corner) near $x=0$ in agreement with Proposition 5.2.

and $\beta_{1}=\beta_{2}=.1, \epsilon_{1}=\epsilon_{2}=10$. As the top row of Figure 6.3, shows, $w_{1}(0)>0$ and $w_{2}(0)=0$ (Proposition 5.2). However, $w_{2}$ is not eliminated from the tube. The bottom left figure shows the steady state solution when the second strain is absent. The bottom right figure shows the steady state solution when the first strain is absent. Notice that the steady state solution $\left(S(x), u_{1}(x), u_{2}(x), w_{1}(x), w_{2}(x)\right)=$ $\left(\bar{S}(x), \bar{u}_{1}(x), 0, \bar{w}_{1}(x), 0\right)$, where $\bar{S}(x), \bar{u}_{1}(x), \bar{w}_{1}(x)$ is the steady state of the first strain in the absence of the second (lower left corner of Figure 6.3) for small $x$.

7. Discussion. In this paper we have investigated a mathematical model of a mixed culture of microorganisms in a tubular flow reactor in which bacteria can colonize the surface of the reactor as well as the bulk fluid. The model, formulated by Freter $[13,14,15]$ for a chemostat setting as a model of the microflora of the gut, can also serve to model the fouling of tubular flow reactors in biotechnology applications. In the case of the human large intestine we argued in [5] that cell motility and nutrient diffusivity were negligible relative to advection, thus motivating our consideration here of plug flow conditions. Indeed, colon length is approximately $150 \mathrm{~cm}$ and realistic transit times of 12-48 hours suggest that $v=5 \mathrm{~cm} / \mathrm{hr}$. Thus, $L / v=30$ hrs compares with a time scale for cell motility $L^{2} / d=112,500 \mathrm{hrs}$ with cell motility coefficients 
$d=0.2 \mathrm{~cm}^{2} / \mathrm{hr}$ relevant to $E$. coli.

As a prelude to our treatment of mixed culture, we showed that a pure culture supplied with a sterile nutrient $\left(u^{0}=0\right)$ can become established if and only if the growth rate of wall-adherent bacteria exceeds the sum of cell death rate and slough-off rates $\left(f_{w}\left(S^{0}\right) G(0)-k_{w}-\beta>0\right)$. This conclusion holds even if free bacteria are unable to grow $(f \equiv 0)$. Numerical simulations indicate that the pure culture steady state, proved to be asymptotically stable in the linear approximation, is globally attracting.

In a mixed culture consisting of two strains, each of which can establish a viable pure culture $\left(f_{w_{i}}\left(S^{0}\right)-k_{w_{i}}-\beta_{i}>0, i=1,2\right)$, it was shown that generically one strain dominates the other at the nutrient-rich, upstream end of the reactor $(x \approx 0)$ in the sense that it has positive density on this segment while the subordinate strain is entirely absent, although it may be present further downstream. It is important to observe that the dominant strain can be predicted from the pure culture steady state densities at $x=0$ : the strain occupying this niche at greatest density in its pure culture steady state is dominant. As mentioned above, this dominance extends to a (possibly small) segment of the upstream end of the reactor. It may extend to the entire reactor. We have identified conditions ensuring that the dominantstrain monoculture solution is locally stable to invasion by the subordinate strain. Of course, this does not ensure that the pure culture solution is globally attracting. We were unable to prove the existence of a stable mixed culture steady state for the case of sterile nutrient supply. However, when the nutrient supply stream includes a constant concentration of each strain $\left(u_{i}^{0}>0, i=1,2\right)$ such a steady state was shown to exist and be stable in the linear approximation. As this case appears to be of lesser significance, we ignore it in the remainder of this discussion.

Numerical simulations of the equations, however, reveal the existence of a stable mixed culture steady state. The most striking result of this paper is the "banded" distribution of the two strains on the wall of the reactor at this steady state (see Figure 6.3). Two strains, differing only in their nutrient uptake characteristics, are seen to establish a stable mixed culture steady state in which the dominant strain is the sole occupant of an upstream band of the reactor while the subordinate strain is essentially the sole occupant of an adjoining band near the upstream end, below which the nutrient density is too low to support either organism. This banded pattern of coexistence occurs when the nutrient uptake curves of the strains cross at an intermediate nutrient density $\left(S^{*}<S^{0}\right)$, when the dominant strain uptake curve exceeds the subordinate strain's for higher nutrient densities $\left(S>S^{*}\right)$, and when the subordinate strain uptake curve exceeds the dominant strain's for lower nutrient densities $\left(S<S^{*}\right)$. As nutrient density $S(x)$ decreases monotonically from the density $S^{0}$ at $x=0$ to a vanishing level further downstream, this banded pattern may be explained heuristically in terms of the well-known competitive exclusion results for two-strain, nutrient-competition in the well-stirred chemostat (see, e.g., [26]). We identify each slice $x=x_{0}$ of the tubular reactor with a well-stirred chemostat supplied with nutrient at concentration $S\left(x_{0}\right)$. At $x_{0} \approx 0, S\left(x_{0}\right)>S^{*}$ so competitive exclusion theory predicts that the dominant strain will eliminate its rival from the chemostat. However, when $x_{0}$ is larger such that $S\left(x_{0}\right)<S^{*}$, the subordinate strain is the one predicted to be dominant in the chemostat and to exclude its rival. Thus the banded structure simply reflects the fact that different dominance relations between pairs of organisms are possible with spatially varying nutrient density.

Numerical simulations with small, but nonzero, cell motility and nutrient diffusivity show that the banded pattern is not an artifact of ignoring diffusion. Therefore, 
this phenomena should be observable in nature or in laboratory environments. We are unaware of any such observations. The human large intestine is, of course, far from being a smooth cylindrical tube and, moreover, it experiences occasional strong peristaltic motion. It is far more likely that the banded structure will be observed in simpler, man-made environments of pipe flow. In these environments, where bacterial contamination is a serious problem, it may be possible in case of contamination by a pathogen to attempt to exclude it by seeding with a more dominant but less pathogenic strain.

In the human large intestine, strong evidence for strain specific wall attachment sites appears to be lacking. It is noted by Wilson in [20],

"As in the stomach and proximal bowel, the wall of the colon is covered with a layer of mucin. The relationship of community members to this thick coating is poorly understood. One would expect that specific adhesion sites would lead to an uneven physical distribution of adherent species."

If our results are applicable to the gut, then they provide another mechanism for spatial segregation of species - namely spatially varying nutrient density which allows for differing dominance relations among a pair of competitors at different locations.

\section{Appendix.}

Proof of Theorem 2.2. As in the Lemma 2.1, let $x_{1}(\tau, s), t_{1}(\tau, s)$ parameterize the characteristics of the first two equations and $x_{2}(\tau, s), t_{2}(\tau, s)$ the last. Thus, given $(x, t) \in \Omega=\left\{(x, t) \in \mathbb{R}^{2} \mid 0<x<1, t>0\right\}$, there exists $s_{1}, s_{2}$ and $\tau_{1}, \tau_{2}>0$ so that $x=x_{1}\left(\tau_{1}, s_{1}\right)=x_{2}\left(\tau_{2}, s_{2}\right), t=t_{1}\left(\tau_{1}, s_{1}\right)=t_{2}\left(\tau_{2}, s_{2}\right)$, and $x_{1}\left(0, s_{1}\right), x_{2}\left(0, s_{2}\right), t_{1}\left(0, s_{1}\right), t_{2}\left(0, s_{2}\right)$ reside on $\partial \Omega$. Along these characteristics

$$
\begin{aligned}
& \frac{d S\left(x_{1}, t_{1}\right)}{d \tau}=-u f(S)-\epsilon w f_{w}(S), \\
& \frac{d u\left(x_{1}, t_{1}\right)}{d \tau}=u(f(S)-k)+\epsilon w f_{w}(S)(1-G(w))-\alpha u(1-w)+\epsilon \beta w, \\
& \frac{d w\left(x_{2}, t_{2}\right)}{d \tau}=w\left(f_{w}(S) G(w)-k_{w}-\beta\right)+\epsilon^{-1} \alpha u(1-w) .
\end{aligned}
$$

At $\tau_{1}=\tau_{2}=0,\left.S\right|_{\tau_{1}=0}=S_{0}\left(s_{1}\right),\left.u\right|_{\tau_{1}=0}=u_{0}\left(s_{1}\right),\left.w\right|_{\tau_{2}=0}=w_{0}\left(s_{2}\right)$ when $t \leq x$, and $\left.S\right|_{\tau_{1}=0}=1,\left.u\right|_{\tau_{1}=0}=u^{0},\left.w\right|_{\tau_{2}=0}=w_{0}\left(s_{2}\right)$ when $x \leq t$.

Let $T>0$, and let $\bar{S}, \bar{u}, \bar{w}$ belong to the space $X:=C\left([0,1] \times[0, T], \Omega_{1}\right)$ with $\Omega_{1}=\left\{(x, y, z) \in \mathbb{R}^{3} \mid 0 \leq x \leq M_{1}, 0 \leq y \leq M_{2}, 0 \leq z \leq 1\right\}$, where $M_{1}, M_{2}$ are defined above Lemma 2.1. We define a metric on $X$ by $\rho((S, u, w),(\bar{S}, \bar{u}, \bar{w}))=$ $\sup |S-\bar{S}|+\sup |u-\bar{u}|+\sup |w-\bar{w}|$, where the supremum is over $[0,1] \times[0, T]$. Then $(X, \rho(\cdot))$ is a complete metric space. To show that (8.1) has a solution we show that the system

$$
\begin{aligned}
& \frac{d S\left(x_{1}, t_{1}\right)}{d \tau}=-u f(S)-\epsilon \tilde{w} f_{w}(S), \\
& \frac{d u\left(x_{1}, t_{1}\right)}{d \tau}=u(f(S)-k)+\epsilon \tilde{w} f_{w}(S)(1-G(\tilde{w}))-\alpha u(1-\tilde{w})+\epsilon \beta \tilde{w}, \\
& \frac{d w\left(x_{2}, t_{2}\right)}{d \tau}=w\left(f_{w}(\tilde{S}) G(\tilde{w})-k_{w}-\beta\right)+\epsilon^{-1} \alpha \tilde{u}(1-w),
\end{aligned}
$$

which defines a map $\mathcal{T}(\tilde{S}, \tilde{u}, \tilde{w})=(S, u, w)$ on $X$, has a fixed point. In particular, we will show that $\mathcal{T}$ is a contraction mapping on $X$. 
We first show that $\mathcal{T}$ maps $X$ into $X$. Under our assumptions the first two equations are a coupled system of ODEs whose vector field is continuous in the $\tau$ variable and globally Lipschitz in the $S, u$ variables. Hence, it has a solution for small enough $\tau$. To see that this solution may be extended for arbitrary $\tau$ notice that the first equation implies that $S\left(x_{1}, t_{1}\right) \geq 0$, since initially $S \geq 0$ and $f(0)=$ $f_{w}(0)=0$. Therefore, Lemma 2.1 implies that $0 \leq S\left(x_{1}, t_{1}\right) \leq M_{1}$ for all $\tau, s$ such that $x_{1}, t_{1} \in[0,1] \times[0, T]$. Similarly, find that $0 \leq S+u \leq M_{2}$, and we obtain existence of $S, u$ for all $\tau, s$ such that $x_{1}, t_{1} \in[0,1] \times[0, T]$. The solution $w$ of the third equation may also be extended for all $\tau$ such that $x_{2}, t_{2} \in[0,1] \times[0, T]$, and clearly $0 \leq w \leq 1$. Moreover, the solution is continuous (in both $\tau$ and $s$ ), and the compatibility conditions, $S_{0}(0)=1, u_{0}(0)=u^{0}$, ensure that the map remains continuous across the interface at $x=t$.

Next we show that the map $\mathcal{T}$ is a contraction of $X$. Let $\left(\tilde{S}_{i}, \tilde{u}_{i}, \tilde{w}_{i}\right) \in X$, $i=1,2$. We need to show that $\sup \left|S_{1}-S_{2}\right|+\sup \left|u_{1}-u_{2}\right|+\sup \left|w_{1}-w_{2}\right| \leq$ $\mu\left(\sup \left|\tilde{S}_{1}-\tilde{S}_{2}\right|+\sup \left|\tilde{u}_{1}-\tilde{u}_{2}\right|+\sup \left|\tilde{w}_{1}-\tilde{w}_{2}\right|\right)$ for some $\mu<1$. The required estimates may be obtained directly from (8.2). For example, by integrating the last equation in (8.2) and using the fact that $\left|f_{w}(\cdot)\right|,|G(\cdot)|,\left|f_{w}^{\prime}(\cdot)\right|,\left|G^{\prime}(\cdot)\right|$ are all uniformly bounded, we obtain

$$
\begin{aligned}
\left|w_{1}-w_{2}\right| \leq & \tau\left[c \sup \left|\tilde{w}_{1}-\tilde{w}_{2}\right|+c \sup \left|w_{1}-w_{2}\right|+c \sup \left|\tilde{S}_{1}-\tilde{S}_{2}\right|\right. \\
& \left.+c \sup \left|\tilde{u}_{1}-\tilde{u}_{2}\right|+M_{2} \alpha \epsilon^{-1} \sup \left|w_{1}-w_{2}\right|\right],
\end{aligned}
$$

where we denote by $c$ a constant that depends only on the parameters $\alpha, \beta, \epsilon, k, k_{w}, M_{1}$ and the bounds on the functions $f, f_{w}, G$ and their derivatives. Thus for $\tau$ sufficiently small, that is $T$ sufficiently small, we find

$$
\sup \left|w_{1}-w_{2}\right| \leq \frac{c T}{1-c T-M_{2} c T}\left[\sup \left|\tilde{S}_{1}-\tilde{S}_{2}\right|+\sup \left|\tilde{u}_{1}-\tilde{u}_{2}\right|+\sup \left|\tilde{w}_{1}-\tilde{w}_{2}\right|\right] .
$$

By adding the first two equations in (8.2) and estimating in the same way, we may find similar estimates on ||$S_{1}-S_{2}|-| u_{1}-u_{2}||$, and then on $\sup \left|S_{1}-S_{2}\right|$ and $\sup \left|u_{1}-u_{2}\right|$. We find that the contraction constant $\left(c+M_{2}\right) T /\left(1-c-M_{2} c T\right)$ may be obtained. Thus for sufficiently small $T$, there exists a $\mu<1$ so that the map $\mathcal{T}$ is a contraction, and we obtain a unique solution to (2.4) on $X$.

Finally we prove that the solutions exist for all $T>0$. We attempt to iterate the above procedure on the space $X:=C\left([0,1] \times\left[T, T^{\prime}\right], \Omega_{1}\right), T^{\prime}>T$. Upon iterating the above procedure the constant $M_{1}$ does not change. However, the constant $M_{2}$ may grow. In fact, the new $M_{2}$ is given by

$$
\sup _{x}\left(M_{1}+u(x, T)+\epsilon \beta, 1+u^{0}+\epsilon \beta\right) .
$$

If the latter term is the largest, $M_{2}$ does not change and we make take $T^{\prime}=2 T$. If the former is largest, we may bound, using the previous bound for $u$, this term by $\sup \left(2 M_{1}+u_{0}(x)+2 \epsilon \beta\right) \leq 2 M_{2}$. We see from the form of the contraction constant that we may take $T^{\prime}=3 T / 2$. In the next iteration we find that our mapping is a contraction of $X:=C\left([0,1] \times[3 T / 2,11 T / 6], \Omega_{1}\right)$. The length of each successive time interval is $T / 2, T / 3 \ldots$. In this way we find that the solution exists for any $T>0$.

Proof of Lemma 3.2. Let $U(s, t)=u(s, t+s)$ and $W(s, t)=w(s, t+s)$ for $0 \leq s \leq 1$ and $t \geq 0$. Integrating the $w$ equation, we get

$$
w(x, t) \leq w_{0}(x) e^{\Lambda t}+C \int_{0}^{t} e^{\Lambda(t-r)} u(x, r) d r,
$$


which leads to

$$
\begin{aligned}
W(s, t) & \leq w_{0}(s) e^{\Lambda(t+s)}+C \int_{0}^{t+s} e^{\Lambda \eta} u(s, t+s-\eta) d \eta \\
& \leq w_{0}(s) e^{\Lambda(t+s)}+C \int_{0}^{t} e^{\Lambda \eta} u(s, t+s-\eta) d \eta+C \int_{t}^{t+s} e^{\Lambda \eta} u(s, t+s-\eta) d \eta \\
& \leq w_{0}(s) e^{\Lambda(t+s)}+C \int_{0}^{t} e^{\Lambda \eta} U(s, t-\eta) d \eta+C \int_{0}^{s} e^{\Lambda(t+s-r)} u(s, \eta) d \eta \\
& \leq e^{\Lambda t} U_{0}(s)+C \int_{0}^{t} e^{\Lambda(t-r)} U(s, r) d r
\end{aligned}
$$

where

$$
U_{0}(s)=w_{0}(s) e^{\Lambda s}+C \int_{0}^{s} e^{\Lambda(s-r)} u(s, \eta) d \eta
$$

Now

$$
\begin{aligned}
\frac{d U}{d s} & \leq A U+B W \\
& \leq A U+B e^{\Lambda t} U_{0}(s)+B C \int_{0}^{t} e^{\Lambda(t-r)} U(s, r) d r
\end{aligned}
$$

which can be integrated $(U(0, t)=0)$ to give

$$
U(s, t) \leq e^{\Lambda t} U_{1}(s)+B C \int_{0}^{s} \int_{0}^{t} e^{-A(s-r)} e^{\Lambda(t-\eta)} U(r, \eta) d \eta d r,
$$

where $U_{1}(s)=B \int_{0}^{s} e^{A(s-r)} U_{0}(r) d r$. Equivalently, if $\bar{V}(s, t)=e^{-\Lambda t} e^{-A s} U(s, t)$, then

$$
\bar{V}(s, t) \leq D+B C \int_{0}^{s} \int_{0}^{t} \bar{V}(r, \eta) d \eta d r
$$

where $D \geq e^{-A s} U_{1}(s)$ for $0 \leq s \leq 1$. A straightforward application of the abstract Gronwall inequality (see page 281 of [28]) implies that $\bar{V}(s, t) \leq V(s, t)$, where $V(s, t)$ is the unique continuous solution of the integral equation

$$
V(s, t)=D+B C \int_{0}^{s} \int_{0}^{t} V(r, \eta) d \eta d r
$$

for $0 \leq s \leq 1$ and $t \geq 0$. The latter exists for $(s, t) \in[0,1] \times[0, T]$ by a simple application of the contraction mapping principle if $B C T<1$ and the solution can be extended to the entire strip $[0,1] \times \mathbb{R}_{+}$by the method of steps.

Putting

$$
V(s, t)=\sum_{n=0}^{\infty} v_{n}(s) t^{n}
$$

into the integral equation and equating powers of $t$ results in

$$
v_{n}(s)=\frac{B C}{n} \int_{0}^{s} v_{n-1}(r) d r, \quad v_{0}(s) \equiv D .
$$


The $v_{n}$ are given by

$$
v_{n}(s)=D \frac{(B C)^{n}}{n !} \frac{s^{n}}{n !}
$$

Hence,

$$
V(s, t)=D \sum_{n \geq 0} \frac{(B C)^{n}}{n !} \frac{s^{n}}{n !} t^{n} \leq P(t),
$$

where $P(t)=D \sum_{n \geq 0} \frac{(B C t)^{n}}{(n !)^{2}}$. But it is easily seen that $e^{-\eta t} P(t)$ is bounded on $[0, \infty)$ for each $\eta>0$. Indeed,

$$
D^{-1} e^{-\eta t} P(t)=\sum_{n \geq 0} \frac{(B C t)^{n} e^{-\eta t}}{(n !)^{2}} \leq 1+\sum_{n \geq 1} \frac{\left(\frac{B C n}{e \eta}\right)^{n}}{(n !)^{2}}<\infty .
$$

Thus

$$
U(s, t)=e^{\Lambda t} e^{A s} \bar{V}(s, t) \leq e^{\Lambda t} e^{A s} V(s, t) \leq e^{(\Lambda+\eta) t} e^{A} e^{-\eta t} P(t) \leq M e^{(\Lambda+\eta) t},
$$

which implies that

$$
u(x, t) \leq M e^{(\Lambda+\eta) t}, \quad t \geq 1 .
$$

Now put this in (8.3) to complete the proof.

Proof of Lemma 4.1. The solution set of $H(S, 0, w)=0$ is easily seen to be as described above using the strict monotonicity of $f_{w}$ and $G$. Hereafter, $S \rightarrow w_{*}(S)$ is the function defined for $0 \leq S \leq 1$ as described above. In case $f_{w}(1) G(0)-$ $k_{w}-\beta<0$ we define $w_{*}(S) \equiv 0$ for $0 \leq S \leq 1$. The lemma essentially follows from a straightforward application of the intermediate value theorem and the implicit function theorem since $H(S, u, 1)=-k_{w}-\beta<0$ and $H\left(S, u, w_{*}(S)\right)>0$ for $0 \leq S \leq 1$ and $u>0$ and $H_{w}<0$ for $0 \leq S \leq 1, u>0, w \geq w_{*}(S)$. Implicit differentiation leads to $h_{S}=-\frac{H_{S}}{H_{w}}$ and $h_{u}=-\frac{H_{u}}{H_{w}}$, and it is easily checked that $H_{S}, H_{u}>0$.

We now focus on the problem of linearized stability of steady state solutions. It is convenient to take a more general point of view. Consider the linear hyperbolic PDE for the vector-valued functions $X$ and $Y$, given by

$$
\begin{aligned}
X_{t}+X_{x} & =a X+b Y, \\
Y_{t} & =c X+d Y, \quad 0 \leq x \leq 1, t>0,
\end{aligned}
$$

with boundary and initial values given by

$$
\begin{aligned}
X(0, t) & =0, \quad t>0, \\
X(x, 0) & =X_{0}(x), \quad Y(x, 0)=Y_{0}(x) .
\end{aligned}
$$

We seek estimates of $X$ and $Y$ on the strip

$$
S \equiv[0,1] \times[0, \infty)
$$

Let $T$ denote the triangular subregion

$$
T=\{(x, t) \in S: x \geq t\}
$$


The following hypotheses will be assumed about the matrices $a, b, c, d$ :

(H1) $a, b, c, d$ are bounded continuous matrix functions on $S$.

(H2) There exists real number $d_{0}$ and $K>0$ such that the fundamental matrix solution $\Psi\left(t, t_{0} ; x\right)$ of the family of initial value problems

$$
\frac{d Y}{d t}=d(x, t) Y, \quad Y\left(t_{0}\right)=I
$$

satisfies the estimate

$$
\left|\Psi\left(t, t_{0} ; x\right)\right| \leq K e^{d_{0}\left(t-t_{0}\right)}, 0 \leq t_{0} \leq t, x \in[0,1] .
$$

The following theorem results.

TheOrem 8.1. Assume that (H1) and (H2) hold. Then for every $\epsilon>0$, there exists $M>0$ such that

$$
|X(x, t)|+|Y(x, t)| \leq M\left[\left\|X_{0}\right\|+\left\|Y_{0}\right\|\right] \exp \left(\left(d_{0}+\epsilon\right) t\right)
$$

holds for $(x, t) \in S$.

Proof. Let $A=\sup _{S}|a(x, t)|$ and similarly $B, C, D$. Use the variation of constants formula for the $Y$-equation to get

$$
Y(x, t)=\Psi(t, 0 ; x) Y_{0}(x)+\int_{0}^{t} \Psi(t, r ; x) c(x, r) X(x, r) d r .
$$

Using (H1) and (H2), we have

$$
|Y(x, t)| \leq e^{d_{0} t}\left\|Y_{0}\right\|+C \int_{0}^{t} e^{d_{0}(t-r)}|X(x, r)| d r .
$$

Now set $U(s, t)=X(s, t+s)$ and $W(s, t)=Y(s, t+s)$ for $0 \leq s \leq 1$ and $t \geq 0$. The equation for $X$ can be written as

$$
\frac{d U}{d s}=a U+b W, \quad U(0, t)=0,
$$

where we must remember that $a, b$ are evaluated along the characteristic. Integrating, we get

$$
U(s, t)=\int_{0}^{s} \Phi(s, \eta ; t) b W(\eta, t) d \eta
$$

where $\Phi(s, \eta ; t)$ denotes the fundamental matrix solution of $\frac{d V}{d s}(s)=a(s, t+s) V(s)$ satisfying $\Phi(\eta, \eta ; t)=I$, which depends on parameter $t$. We have the estimate

$$
|\Phi(s, \eta ; t)| \leq e^{A(s-\eta)} \leq K=e^{A}
$$

for $0 \leq \eta \leq s \leq 1$ and $t \geq 0$. Hence, we have

$$
|U(s, t)| \leq K B \int_{0}^{s}|W(\eta, t)| d \eta .
$$


Using (8.4) to estimate $W(s, t)$ as below (8.3) in the proof of Lemma 3.2, we have

$$
|W(s, t)| \leq e^{d_{0} t} U_{0}(s)+C \int_{0}^{t} e^{d_{0}(t-r)}|U(s, r)| d r,
$$

where

$$
\begin{aligned}
U_{0}(s) & =\left\|Y_{0}\right\| e^{d_{0} s}+C \int_{0}^{s} e^{d_{0}(s-r)}|X(s, r)| d r \\
& \leq L\left[\left\|Y_{0}\right\|+C \max _{T}|X(x, t)|\right]
\end{aligned}
$$

with $L=e^{d_{0}}$. Inserting this estimate for $W$ into the inequality for $U$ gives

$$
\begin{aligned}
|U(s, t)| & \leq K B \int_{0}^{s}\left[e^{d_{0} t} U_{0}(\eta)+C \int_{0}^{t} e^{d_{0}(t-r)}|U(\eta, r)| d r\right] d \eta \\
& \leq K B e^{d_{0} t}\left[L\left(\left\|Y_{0}\right\|+C \max _{T}|X(x, t)|\right)+C \int_{0}^{s} \int_{0}^{t} e^{-d_{0} r}|U(\eta, r)| d r d \eta\right] .
\end{aligned}
$$

Setting $V(s, t)=e^{-d_{0} t}|U(s, t)|$, we have

$$
V(s, t) \leq K B\left[L\left(\left\|Y_{0}\right\|+C \max _{T}|X(x, t)|\right)+C \int_{0}^{s} \int_{0}^{t} V(\eta, r) d r d \eta\right] .
$$

By the proof of Lemma 3.2 (note the factor $D$ in the definition of function $P(t)$ there), given $\epsilon>0$, there exists $M>0$ such that

$$
V(s, t) \leq M K B\left[L\left(\left\|Y_{0}\right\|+C \max _{T}|X(x, t)|\right)\right] e^{\epsilon t},
$$

which implies that

$$
|U(s, t)| \leq M K B\left[L\left(\left\|Y_{0}\right\|+C \max _{T}|X(x, t)|\right)\right] e^{\left(d_{0}+\epsilon\right) t}
$$

for $0 \leq s \leq 1, t \geq 0$, and therefore

$$
|X(x, t)| \leq M K B\left[L\left(\left\|Y_{0}\right\|+C \max _{T}|X(x, t)|\right)\right] e^{\left(d_{0}+\epsilon\right) t}
$$

for $(x, t) \in S$ with $t>x$.

Similar estimates of $X$ and $Y$ in region $T$ lead to the estimate

$$
\max _{T}|X(x, t)| \leq P\left(\left\|X_{0}\right\|+\left\|Y_{0}\right\|\right)
$$

for suitable constant $P>0$. Putting these estimates together and using (8.4) we get the desired result.

COROLLARY 8.2. If the continuous matrix function d depends only on $x \in[0,1]$ and not on $t$ and if it is a stable matrix for each $x \in[0,1]$, then (H2) holds with $d_{0}<0$. In this case, we may take $\epsilon<-d_{0}$ in the theorem in order to conclude the exponential asymptotic stability of the PDE.

Proof. Use Proposition 3 in Chapter 1 of [9] and the continuity of $d$.

It should be noted that asymptotic stability in the linear approximation does not automatically mean asymptotic stability for the nonlinear problem, although it is usually a first step in stability analysis. We defer to a later work a proof of asymptotic stability. 


\section{REFERENCES}

[1] H. Amann, Fixed point equations and nonlinear eigenvalue problems in ordered Banach spaces, SIAM Rev., 18 (1976), pp. 620-709.

[2] J. Bailey And D. Ollis, Biochemical Engineering Fundamentals, 2nd ed., McGraw-Hill, New York, 1986.

[3] M. Ballyk, L. Dung, D. A. Jones, And H. L. Smith, Effects of random motility on microbial growth and competition in a flow reactor, SIAM J. Appl. Math., 59 (1999), pp. 573-596.

[4] M. Ballyk And H. L. Smith, A flow reactor with wall growth, in Mathematical Models in Medical and Health Sciences, M. Horn, G. Simonett, G. Webb, eds., Vanderbilt University Press, Nashville, TN, 1998.

[5] M. Ballyk And H. L. Smith, A model of microbial growth in a plug flow reactor with wall attachment, Math. Biosciences, 158 (1999), pp. 95-126.

[6] B. Baltzis And A. G. Fredrickson, Competition of two microbial populations for a single resource in a chemostat when one of them exhibits wall attachment, Biotech. Bioengrg., 25 (1983), pp. 2419-2439.

[7] A. Berman and R. J. Plemmons, Nonnegative Matrices in the Mathematical Sciences, Academic Press, New York, 1979.

[8] W. G. Characklis and K. C. Marshall, eds., Biofilms, Wiley Series in Ecological and Applied Microbiology, John Wiley and Sons, New York, 1990.

[9] W. Coppel, Dichotomies in Stability Theory, Lecture Notes in Math. 629, Springer-Verlag, New York, 1978.

[10] J. W. Costerton, Z. Lewandowski, D. DeBeer, D. Caldwell, D. Korber, and G. James, Minireview: Biofilms, the customized microniche, J. Bacteriology, 176 (1994), pp. 21372142.

[11] J. W. Costerton, Z. Lewandowski, D. Korber, and H. M. Lappin-Scott, Microbial biofilms, Ann. Rev. Microbiology, 49 (1995), pp. 711-745.

[12] Le Dung And H. L. Smith, A parabolic system modeling microbial competition in an unmixed bio-reactor, J. Differential Equations, 130 (1996), pp. 59-91.

[13] R. Freter, H. Brickner, J. Fekete, M. Vickerman, and K. Carey, Survival and implantation of E. coli in the intestinal tract, Infection and Immunity, 39 (1983), pp. 686-703.

[14] R. Freter, Mechanisms that control the microflora in the large intestine, in Human Intestinal Microflora in Health and Disease, D. Hentges, ed., Academic Press, New York, 1983.

[15] R. Freter, H. Brickner, And S. Temme, An understanding of colonization resistance of the mammalian large intestine requires mathematical analysis, Microecology and Therapy, 16 (1986), pp. 147-155.

[16] S. B. Hsu, H. L. Smith, and P. Waltman, Competitive exclusion and coexistence for competitive systems on ordered Banach spaces, Trans. Amer. Math. Soc., 348 (1996), pp. 40834094.

[17] F. John, Partial Differential Equations, Springer-Verlag, New York, 1971.

[18] C.-M. Kung AND B. BALTZIS, The growth of pure and simple microbial competitors in a moving and distributed medium, Math. Biosci., 111 (1992), pp. 295-313.

[19] A. LeE, Neglected niches. The microbial ecology of the gastrointestinal tract, in Advances in Microbial Ecology, K. C. Marshall, ed., Plenum Press, New York, 1985.

[20] R. Mackie, B. White, and R. Isaacson, eds., Gastrointestinal Microbiology, Vol. 2, Chapman and Hall, NY, 1997.

[21] X. Mora, Semilinear Parabolic problems define semiflows on $C^{k}$ spaces, Trans. Amer. Math. Soc., 278 (1983), pp. 21-55.

[22] H. Matano and M. Mimura, Pattern formation in competition-diffusion systems in nonconvex domains, Pub. Res. Inst. Math. Sci. Kyoto Univ., 19 (1983), pp. 1050-1079.

[23] S. S. Pilyugin and P. Waltman, The simple chemostat with wall growth, SIAM J. Appl. Math., 59 (1999), pp. 1552-1572.

[24] H. L. Smith, Monotone Dynamical Systems: An Introduction to the theory of competitive and cooperative systems, AMS Math. Surv. Monogr., 41, Providence, RI, 1995.

[25] H. Topiwala And G. Hamer, Effect of wall growth in steady state continuous culture, Biotech. Bioengrg., 13 (1971), pp. 919-922.

[26] H. L. Smith and P. Waltman, The Theory of the Chemostat, Cambridge University Press, Cambridge, UK, 1995.

[27] H. Thieme, Persistence under relaxed point-dissipativity (with application to an epidemic model), SIAM J. Math. Anal., 24 (1993), pp. 407-435.

[28] E. ZeIdLeR, Nonlinear Functional Analysis and Its Applications, I, Springer-Verlag, New York, 1993. 